\title{
ABSCESO RECIDIVANTE POR NOCARDIA ASTEROIDES EN UNA PACIENTE PORTADORA DE POLIGLOBULIA PRIMARIA
}

\author{
Carlos Aboal', Ronald Salamano², Adelina Braselli, \\ Mariel Mansilla ${ }^{4}$, Maria Galaret ${ }^{5}$, Walter Pedreira ${ }^{6}$
}

\begin{abstract}
RESUMEN - La nocardiosis del sistema nervioso central (SNC) es una enfermedad poco común, cuya frecuencia ha aumentado con el crecimiento de los tratamientos inmunosupresores. El hombre se infecta por inhalación, inoculación traumática cutánea directa y tras una infección periodontal ingiriendo alimentos contaminados. La localización pulmonar es la más frecuente, siendo la fuente inicial de diseminación hemática, con p redominio en piel, tejido celular subcutáneo y SNC. Es conocida la predilección de Nocardia por el SNC. Estos abscesos, asociados a altos índices de mortalidad, especialmente en pacientes inmunocomprometidos, siguen constituyendo un desafío diagnóstico y terapéutico, permaneciendo no aclarado su manejo terapéutico óptimo. A pesar de la controversia en cuanto al manejo quirúrgico de estas lesiones, el diagnóstico precoz, por aspiración esterotáxica, y la iniciación de una terapia antimicrobiana son esenciales para la buena evolución del paciente. Se presenta una paciente portadora de una poliglobulia primaria, con absceso cerebral recidivante por Nocardia asteroides de posible origen dentario.
\end{abstract}

PALABRAS CLAVES: absceso cerebral, nocardia, poliglobulia primaria.

\begin{abstract}
Recument brain abscess caused by Nocarda asteroides in a patient with primary polycythaemia
ABSTRACT - Nocardiosis of the central nervous system (CNS) is an uncommon disease, but its frequency has increased due to the high number of immunosuppressive treatments. People become infected by inhalation, direct traumatic cutaneous inoculation and eating contaminated food after a periodontal abscess. Lung localization is the most frequent one, being the origin of haematic dissemination, with a high incidence in skin, subcutaneous tissue and the CNS. The prefe rence of Nocardia for the CNS is well-known. These abscesses are a diagnostic and therapeutic challenge, since they are associated to high mortality rates, specially in immunocompromised patients; the best therapeutic management remains unclear. In spite of the existing controversy with regard to the surgical management of these lesions, an early diagnosis th rough stereotactic aspiration and the beginning of an antimicrobial therapy are essential to the patient's good evolution. This paper presents a patient with a primary polycythaemia and a re cu rrent brain abscess by Nocardia asteroides, probably of dental origin.
\end{abstract}

KEY WORDS: brain abscess, nocardia, primary polycithaemia.

Con el término de nocardiosis se designa a la enfermedad invasiva, localizada o diseminada, ocasionada por especies del género Nocardia.

La nocardiosis del sistema nervioso central (SNC), es una enfermedad poco común, pero su fre c u e n ci a ha aumentado últimamente después del use agresivo de la quimioterapia para el tratamiento de enfermedades malignas y el importante aumento en la población de personas transplantadas sometidas a tratamientos inmunosupresores. El aumento de inciden- cia también puede reflejar las mejores posibilidades diagnósticas y la mayor búsqueda de esta etiología vinculada al mayor índice de sospecha'.

Nocardia es una bacteria aerobia estricta, filamentosa, ramificada, Gram positiva, de crecimiento lento, por lo que cuando se sospecha la enfermedad el laboratorista debe estar advertido para no desechar los medios sembrados antes de los 14 días y pueden ser necesarias hasta 4 semanas para que el germen alcance su forma característica. Pertenece a la

\footnotetext{
1Profesor Adjunto de Neurocirugía, Instituto de Neurología, Facultad de Medicina, Montevideo, Uruguay (IN/FM); ${ }^{2}$ Profesor Agregado de Neurología, IN/FM; ${ }^{3}$ P rofesor de Infectología, Servicio de Enfermedades Infectocontagiosas; ${ }^{4}$ Profesor Agregado de Infectología, Servicio de Enfermedades Infectocontagiosas; ${ }^{5}$ Profesor Adjunto de Neurocirugía, Facultad de Medicina; ${ }^{6}$ Bacteriólogo.
}

Received 14 September 2005, received in final form 16 January 2006. Accepted 24 February 2006.

Dr. Carlos Aboal - Instituto de Neurología - $2^{\circ}$ piso - Hospital de Clínicas Dr. Manuel Quintela - Avenida Italia s/n - Montevideo 11600 
familia Nocardiaceae, la que se incluye en el orden de los Actinomicetales. Nocardia dela especieaste roides es la responsable de la mayor parte de los casos de enfermedad pulmonar diseminada. Otras, como $N$. brasiliensis es la causa principal de lesiones cutáneas'.

Tiene una distribución universal y es encontrada en el suelo, agua, aire y vegetales contaminados. Fue identificada por primera vez en 1888 por Edmond Nocaro ${ }^{2,3}$ y dos años más tarde Eppinger ${ }^{4}$ describió el primer caso humano de absceso cerebral ${ }^{3,4}$.

El hombre se infecta a través del aparato respiratorio por inhalación; por inoculación traumática cutánea directa, y tras una infección periodontal al ingerir alimentos contaminados. No ha sido demostrada la transmisión interhumana ni de animales a personas. Aunque con relativa frecuencia afecta a personas inmunocompetentes, se la considera una enfermedad o p o rtunista en personas que presentan algún factor d e te rminante de inmunodepresión, ya sea local (enf e rmedad pulmonar obstructiva crónica, bronquiectasias) o sistémica. Se le observa en personas con enfe rmedades hematooncológicas malignas, receptores de transplante de órganos sólidos, alcohólicos, cirr óticos, diabéticos, portadores de colagenopatías o tratados crónicamente con corticoides ${ }^{1,3,5-7}$. Raramente fue descrita en pacientes infectados VIH lo que se atribuye a la profilaxis que se realiza en estos pacientes de la infección por Pneumocystis carinii con trimetoprin sulfametoxasol (TMP/SMX) o al tratamiento que reciben con sulfas frente a imágenes en forma de anillo que captan contraste, por la eventualidad de una neurotoxoplasmosis 5 . La enfermedad suele presentarse en forma crónica o subaguda y ocasionalmente aguda en inmunocomprometidos.
La localización pulmonar es la más frecuente, siendo la fuente inicial de diseminación hemática en diferentes localizaciones, aunque con predominio en piel, tejido celular subcutáneo y sistema nervioso central (SNC). Es conocida la característica predilección que tiene Nocardia por invadir el SNC 1,5 . Entre mil cincuenta casos de nocardiosis seleccionados al azar de la literatura, 22,7\% de los pacientes desarrollaron subsiguientes infecciones secundarias del SNC'. Mamelak et al. han estimado que el riesgo puede llegar a $45 \%^{5-8}$. Dado el carácter silente que pueden tener las localizaciones neurológicas y su elevada frecuencia, se aconseja realizar TAC y/o RM de cráneo siempre que se diagnostica nocardiosis pulmonar. La meningitis es mucho menos frecuente que las lesiones encefálicas, las que pueden presentarse como infiltración cerebral difusa, seudoinfartos y abscesos. Estos pueden ser únicos o múltiples y con más frecuencia son supratentoriales. Raramente se han documentado abscesos medulares ${ }^{5,6,8}$.

Del total de abscesos cerebrales solo $2 \%$ son causados por Nocardia. Sin embargo tienen tres veces más mortalidad que los abscesos de otras etiologías, especialmente en los enfermos inmunodeprimidos. La literatura publicada es limitada y con reporte de pocos casos, lo que hace difícil establecer conclusiones sobre el óptimo tratamiento médico y quirúrgico

Motiva la presente comunicación el tratarse de un caso clínico excepcional por corresponder a una fo rma recidivante de nocardiosis encefálica, estando el segundo episodio muy distanciado en el tiempo respecto al primero, ambos precedidos de una maniobra odontológica y asentar en una paciente portadora de poliglobulia primaria.

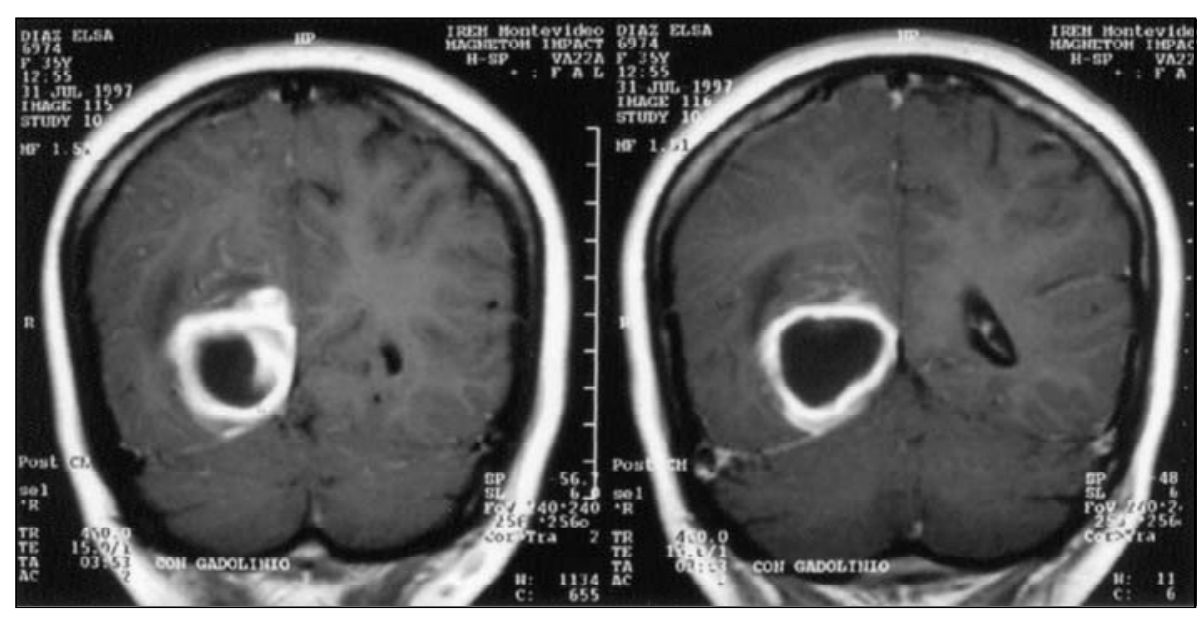

Fig 1. RM ponderada en $T 1$ con gadolinio en plano coronal. Evidencia proceso expansivo cortico-subcortical con anillo gruesco irregular de realce con centro necrótico. 


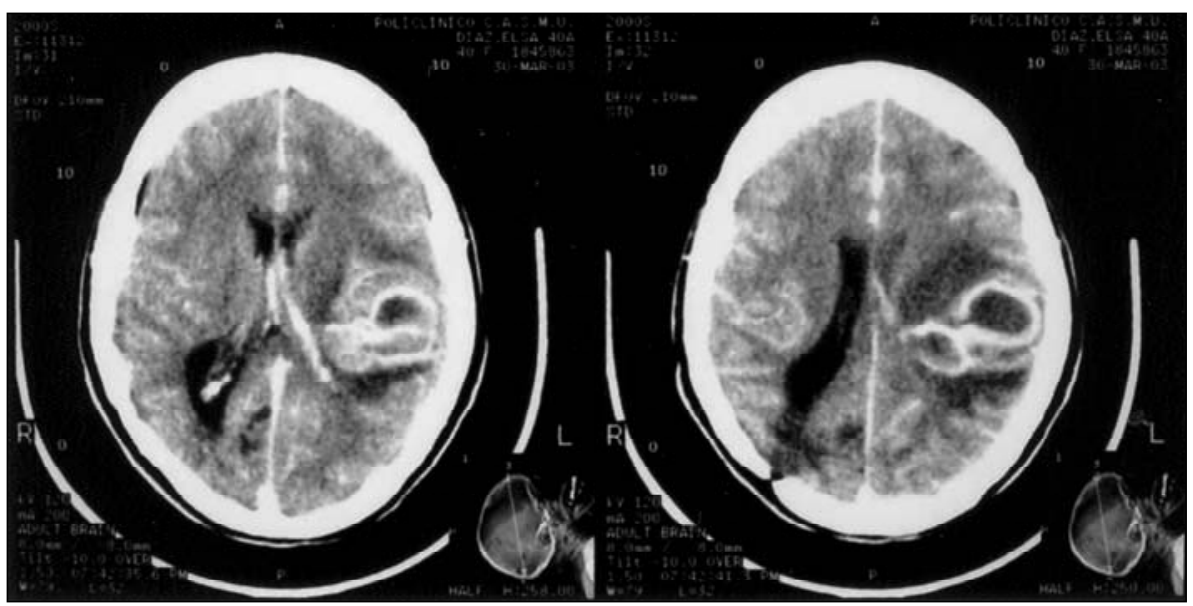

Fig 2. TC con medio de contraste. Evidencia proceso expansivo parietal izquiedo con realce anular de aspecto multiloculado.

\section{CASO}

Mujer de 42 años, diestra, portadora de poliglobulia diagnosticada 15 años antes y catalogada como primaria luego de exhaustivos estudios. Tratada con ácido acetil salicílico en las ocasiones que se le realizó flebotomías, tuvo mala tolerancia a las mismas. A principios de julio de 1997 fue sometida a un procedimiento de extracción e implante dentario, presentando 10 a 15 días después un cuadro de inicio y pro g resión insidiosos, en el curso de 15 días, y caracterizado por cefalea, vómitos, fiebre, a lo que se agre$\mathrm{g}$ a ron posteriormente alteraciones de la agudeza visual y campimétricas y en las últimas cuarenta y ocho horas, depresión de la vigilia. En el examen físico, la paciente se presentaba confusa, por momentos excitada, con guiño a la amenaza abolido a izquierda, paresia de VI par izquierdo, parálisis facial central izquierda, paresia leve de miembros izquiercbs, con hiporreflexia y signo de Babinski. El re s to del examen general fue normal. La RM con gadolinio evidencia proceso expansivo cortico-subcortical con anillo grue so irregular de realce con centro necrótico (Fig 1). La orientación diagnóstica imagenológica RM orientó a un absceso cerebral o glioblastoma multiforme. En la paraclínica de laboratorio el hematocrito fue de $6,79 \%$, hemoglobina 20,9 mg/dL, VCM 90,1, HBCM 30,7\%, leucocitos $12,800 / \mathrm{mm} 3$, PMN $80 \%$, linfocitos $17 \%$, eosinófilos $1 \%$, basófilos $2 \%$, plaquetas $150 / \mathrm{mm} 3$, VES $1 \mathrm{~mm} 1^{\text {a }}$ hora, PEF normal. El ECG transesofágico fue normal y la serología para VIH negativa. La radiografía de tórax fue normal y los hemocultivos negativos. La paciente fue intervenida quirúrg icamente de urgencia, realizándose drenaje de la colección supurada. El material extraído se envió para estudio microbiológico aislándose Nocardia asteroides. La paciente fue tratada con TMP/SMX a la dosis de $3.200 / 640 \mathrm{mg} / \mathrm{d}$ en dos dosis, por nueve meses. Como segundo antibiótico se usó ceftriaxona $2 \mathrm{~g} / \mathrm{d}$ i/v c/12 horas durante el primer mes. Los contrdes clínicos e imagenológicos mostraron evolución favorable, quedando la enferma asintomática.

En marzo de 2003 y después de un nuevo procedimiento de extracción e implante dentario (90 días), ingresó al sanatorio por un cuadro de comienzo agudo y rápidamente evolutivo, caracterizado por cefalea, vómitos, fiebre de 39$40^{\circ} \mathrm{C}$, alteraciones del habla, guiño abolido a derecha y hemiparesia derecha con signo de Babinski. El examen físico general fue normal. La TC de cráneo mostró un proceso expansivo parietal izquierdo de cuatro centímetros de diámetro, imagen ligeramente redondeada que serealzaba con el medio de contraste, con edema perilesional (Fig 2). El aspecto era de absceso encefálico. Las TC de tórax y abdomen fueron normales. Intervenida quirúrgicamente se d renó la colección purulenta. En el cultivo de este material se identificó Nocardia asteroides. El plan de antibióticos iniciado fue el mismo que el anterior, prolongándose la ceftriaxona por seis meses y el TMP/SMX por un año. La evolución fue favorable, manteniéndose asintomática hasta el último control que fue en diciembre de 2004.

\section{DISCUSION}

Los abscesos cerebrales nocardiales siguen constituyendo un desafío diagnóstico y terapéutico, estando asociados a altos índices de mortalidad, especialmente en pacientes inmunocomprometidos ${ }^{1,5,8,9}$. La localización supratentorial es más frecuente que la infratentorial. En $54 \%$ de los casos son lesiones únicas y en $38 \%$ son lesiones múltiples ${ }^{5,9}$. Una revisión de 120 casos re po rtados en la literatura desde 1950 demostraroníndices de mortalidad de $24 \%$ luego de la craniotomía y escisión iniciales, 50\% luego de la aspiración-drenaje y $30 \%$ luego de terapia antimicrobiana en exclusiva. Más importante aún, $22 \%$ de los casos fueron diagnosticados en la necropsia. El índice de mortalidad fue $33 \%$ en pacientes con un solo absceso y se duplicó cuando los abscesos eran múltiples. $34 \%$ de los pacientes eran inmunocomprometidos, $40 \%$ de estos eran transplantados y solo $15 \%$ eran VIH positivos. Es decir que solo $5 \%$ de los pacientes 
con absceso por Nocardia eran VIH positivos. La mortalidad en los inmunocomprometidos es bastante mayor ( $55 \%$ vs. $20 \%$, en la era tomográfica) $)^{5,9}$. Varias publicaciones recientes no ponen de manifiesto alta mortalidad, pero ello puede ser consecuencia de sesgo en la selección de las muestras ${ }^{3}$.

Los pacientes con nocardiosis sistémica se presentaron en $15-44 \%$ de los casos estudiados ${ }^{1,3}$. Sin emb a rgo, los abscesos por nocardia constituyen sólo $2 \%$ de los abscesos cerebrales ${ }^{3}$. Es frecuente que haya múltiples lesiones en el mismo individuo. Cuando ocurren abscesos solitarios, estos son con frecuencia multiloculados. Aún cuando se ha sugerido que los abscesos nocardiales con frecuencia se hallan pobremente encapsulados, una cápsula definida fue identificada en algunos casos. Una fuente extraneurológica de la infección se pudo identificar en $66 \%$ de los casos, siendo el compromiso pulmonar el más común. Un tercio de los pacientes publicados en la literatura tenían algún factor de inmunocompromiso $0^{3,5}$. En segundo lugar, con frecuencia no causan fiebre ni aumento de la leucocitosis que puedan alertar a los clínicos sobre la posibilidad de una infección bacteriana, en consecuencia y atendiendo además a la imagenología muchos casos pueden diagnosticarse erróneamente como tumores malignos cerebrales".

Asimismo, el manejo óptimo de los abscesos cerebrales por Nocardia pe rmanece poco claro. La serie quirúrgica con más pacientes fue de once tratados en diferentes hospitales 5 . El resto de las comunicaciones son de casos aislados o de pocos pacientes. A pesar de la limitada literatura, la escisión quirúrgica aparece como la principal alternativa terapéutica. Lee et al. ${ }^{9}$ aludieron a la posibilidad de tratar estas lesiones sólo con aspiración. Sin embargo esta recomendación es consecuencia de tres pacientes operados, uno de ellos sometido posteriormente a una craniotomía para una solución definitiva de su absceso. Este hallazgo sugiere que las aspiraciones quirúrgicas repetidas deberían considerarse apropiadas sólo como tratamiento inicial, especialmente en pacientes con lesiones profundas y/o múltiples. El único paciente que exhibió deterioro neurológico luego de una aspiración simple y que requirió cirugía abierta fue una paciente con un absceso grande multilocular temporal.

A pesar de la controversia en lo que se refiere al manejo quirúrgico de estas lesiones, está claro que el diagnóstico temprano, por la vía de la aspiración este rotáxica, y la iniciación de una terapia antimicrobiana apropiada son esenciales. Las sulfonamidas solas o combinadas en trimetoprim son efectivas y los tratamientos deben prolongarse por la frecuente recidiva. La minociclina, el imipenem o los aminoglucósidos en colaboración con una cefalosporina de t e rcera generación, pueden ser utilizados con éxito como tratamiento de segunda línea en caso de alergias o no respuesta a las sulfas. La duración óptima de la terapia es desconocida, aun cuando se favorece generalmente el tratamiento a largo plazo debido a la tendencia a la recaída ${ }^{1,5}$. Una terapia por el curso de 12 meses se recomienda actualmente para el tratamiento de los abscesos cerebrales por Nocardia. Incluso períodos mas largos de tratamiento pueden ser necesarios para los individuos inmunocomprometidos, aún cuando los regimenes de tratamiento deben ser individualizados ${ }^{1}$.

Nuestrocaso clínico reviste la singularidad de no haber encontrado en la literatura internacional revisada la asociación con la poliglobulia esencial ni tampoco la recidiva de los abscesos luego de un lapso tan prolongado. El tratamiento en las dos oport u $\mathrm{n}$ idades fue de abordaje directo con drenaje y escisión mas antibióticoterapia adecuada (cefalosporina de tercera generación más trimetoprim-sulfametoxasol) en forma prolongada (nueve meses y un año). Pensamos que no hubo esterilización del foco inicial (dentario) por lo que frente a una nueva manipulación se reprodujo la bacteriemia y localización.

\section{REFERENCIAS}

1. Mandell W, Neu HC. Nocardial infections. In Vinken PJ, Bruyn GW, Klawans HL, (eds). Handbook of Clinical Neurology Vol. 8. Microbial Disease. Amsterdam: Elsevier, 1988;445-453.

2. NocardME. Note sur la maladie des boeufs de la Guadaloupe: connue sous le nom de farcin. Ann Inst Pasteur Microbiol 1888;2:293-307.

3. Fleetwood IG, Embil JM, Ross I B. Nocardia asteroides cerebral abscess immunocompetent hosts: report of three cases and review of surgical recommendations. Surg Neurol 2000;53:605-610.

4. Eppinger $\mathrm{H}$. Ueber eine neue, pathogene Cladothrix and eine durch die hervorg e rufene Pseudotuberculosis (Cladothichica). Beitr Pathol Anat 1890;9:287-328.

5. Mamelak AN, Obana WG, Flaherty JF, Rosemblum ML. Nocardial brain abscess: treatment strategies and factors infuencing outcome. Neurosugery 1994;35:622-631.

6. Born W, Gleixner M. Nocardia brain abscess misinterpreted as cerebral infarction. J Clin Neurosci 2003;10:130-132.

7. Hueb Barata C, Alvarenga Gomes Oliveira D, Lopes Colombo A, Pires Pereira CA. Absceso cerebral por Nocardia sp. en paciente inmunosuprimido. Rev Soc Bras Med Trop 2000;33:609-612.

8. Durmaz R, Atasoy M A, Durmaz G, et al. Multiple nocardial abscesses of cere b rum, cerebellum and spinal cord, causing quadriplegia. Clin Neurol Neurosurg 2001;103:59-62.

9. Lee GYF, Daniel RT, Brophy BP, Reilly PL. Surgical treatment of nocardial brain abscesses. Neurosurgery 2002;51:668-671. 